\title{
HIPOTESIS CAUSALES SOBRE FECUNDIDAD: \\ EL PAPEL PREPONDERANTE DE LA \\ UNIDAD FAMILIAR
}

Josune Aguinaga Roustán

La preocupación que existe a nivel mundial acerca de la problemática de la población discurre a través de dos vertientes aparentemente contradictorias. La primera de ellas hace referencia a la superpoblación mundial: las Naciones Unidas estiman que para el año 2000 la población del planeta superará los $6.000 \mathrm{mi}$ llones, existiendo fundadas dudas de que para esta población exista un nivel de subsistencia mínimo; la segunda se refiere al descenso secular de la fecundidad, que en algunos países no alcanza ya el nivel de reemplazo o, lo que es lo mismo, el nivel de crecimiento de la población está por debajo de cero.

Resolver esta contradicción es tarea simple, en el primer caso hemos hablado de «población mundial» y en el segundo de algunos países, así vemos que el crecimiento poblacional se compone de la agregación comportamental de dos grandes núcleos de población: países subdesarrollados, con altas tasas de natalidad, y paises desarrollados, con bajas tasas de natalidad.

Ocurre que mientras los primeros concentran aproximadamente un 75 por 100 del total de la población mundial, los segundos apenas alcanzan el 25 por 100 restante. El resultado de esta situación es que la población mundial sigue creciendo al tiempo que la relación entre unos y otros va inclinándose en favor de los subdesarrollados. En unos pocos años, y mientras continúen las restricciones sobre la inmigración, la proporción de población en los países desarrollados estará por debajo del 20 por 100 .

Los factores estructurales que diferencian ambos tipos de crecimiento demográfico y los objetivos últimos de los trabajos que analizan la fecundidad hacen que los mismos, a pesar de partir de una metodología común, esto es, un análisis de 
las variables que inciden sobre la fecundidad, presenten caracterísicas muy diferenciadas.

Ya que en el caso español nos encontramos, demográficamente, en un país desarrollado, o en permanente transición hacia el desarrollo, vamos a tratar aquí las teorías de la fecundidad en los países desarrollados, las cuales han ido evolucionando en el sentido de ir aislando variables y profundizar en ellas, lo que ha dado lugar a una ingente cantidad de estudios empíricos como pasos previos para obtener una teoría explicativa de la fecundidad.

La fecundidad ha sido analizada desde diversas disciplinas, cada una de las cuales ha sido predominante en un período histórico determinado, entre ellas destacan la biología, la economía, la demografía, la sociología, la psicología, etc., de las que me ocupo someramente a continuación.

La biología, tan preponderante en etapas anteriores, ha quedado ya obsoleta como consecuencia de intentar explicar la fecundidad prescindiendo de factores socioeconómicos y culturales, por ejemplo, una de las variables más manejadas por esta disciplina, la alimentación, no nos ofrece ninguna salida si la utilizamos como una especie de concepto objetivo e independiente; sin embargo, se está produciendo una reentrada de factcres biológicos en la perspectiva de factores culturales, como más adelante veremos. En este aspecto podemos añadir que estamos pendientes de los aportes de la sociobiología que, según Wilson, nos proporcionará a los sociólogos un "conjunto duradero de principios primarios»'. aunque hasta ahora y en el campo de la fecundidad no haya aportado nada.

En cuanto a la economía, es la disciplina que tiene una mayor tradición respecto a los estudios de la población, centrándose ésta en la polémica entre malthusianos y antimalthusianos, y en el interés que la cuestión de la población activa ofrece para los economistas. Además, los especialistas en historia económica se han ocupado muy ampliamente de los aspectos demográficos. Sin embargo, en 1960, los economistas se encontraban en un callejón sin salida, ya que no habían conseguido ningún avance teórico importante; es justo en estos años cuando comienza a desarrollarse la llamada "nueva economía doméstica»; cuyas valiosas aportaciones serán ampliamente analizadas más adelante.

El problema de la demografía es más complejo, no cabe duda de que los demógrafos llevan años, por no decir siglos, trabajando sobre los datos de la población, su labor es imprescindible, pero estoy de acuerdo con los términos en los que un conocido demógrafo ha planteado el problema: «... esto significa que si los demógrafos desean explicar el fenómeno que estudian deben o bien convertirse en auténticos científicos sociales o alternativamente confinarse a medir lo que preguntan otras disciplinas para explicarlo» ${ }^{2}$. Es decir, la demografía y las téc-

' Wuson, Edward O., Sociobiologia. La nueva sintesis, Barcelona: Omega, 1980, página 593. El intento realizado en este sentido por el mismo Wilson, de sistematizar las aportaciones de la biologia en el campo de la sociologia, creo que ha terminado en un relativo fracaso, que es el de toda la Sociobiología, en el campo de las explicaciones causales, que pudieran servir para explicar la fecundidad. Véase WiLson, E. 0 ., Sobre la naturaleza humana, Madrid: Fondo de Cultura Económica, 1983.

2 Caldwell, S. E., "The Mechanisms of Demographic Change in Historical Perspective", Population Studies, vol. 35, núm. 1, marzo 1981, p. 25. 
nicas demográficas son imprescindibles para todas las disciplinas que traten de problemas de población, y al mismo tiempo si la demografía pretende pasar a niveles explicativos está en el campo de una de esas disciplinas. En este sentido, Amando de Miguel realiza una afirmación similar: «Los sociólogos debemos aportar nuestro punto de vista al análisis de las poblaciones, lo que equívoca y equivocadamente se ha dado en llamar 'demografía', como si fuese una ciencia aislada y estanca, y no una corriente que baña todas las ciencias sociales» ${ }^{3}$.

En relación con la sociología, lo mismo que con la psicología social, el interés por el tema de la fecundidad ha resultado fruto de un camino indirecto como consecuencia de la utilización de métodos, técnicas y explicaciones sociológicas por parte de los practicantes de otras disciplinas, lo que de rechazo ha conducido a que algunos sociólogos se interesaran por tales temas, como luego veremos, la entrada de la sociología en el campo de la fecundidad es muy reciente, pero de resultados esperanzadores.

Paso a continuación a presentar las teorías concretas sobre la fecundidad que se han desarrollado desde las mencionadas disciplinas.

A pesar del tiempo transcurrido, la presencia de Malthus sigue condicionando el contexto en el que se desarrollan las diversas teorías, que reinterpretan o rechachan al clérigo inglés de acuerdo con el contenido particular de su teoría, por ejemplo, en el apartado correspondiente de la «nueva economía doméstica» de la fecundidad, leemos, por un lado: «una manera de avanzar hacia una teoría de la población que sea compatible con la experiencia del mundo occidental y al mismo tiempo coherente con la teoría económica, consiste en reexaminar la teoría mal-

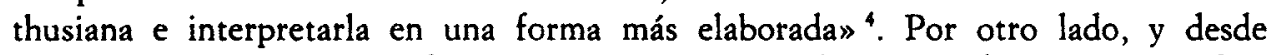
una perspectiva opuesta, se lee: «Impotentes para explicar estos fenómenos, muchos científicos sociales han rechazado la teoría malthusiana como relevante en la actualidad, y han querido buscar en otra parte una teoría de la fecundidad» 5 .

Al inicio de la década de los 60 , en la escuela de microeconomía de Chicago, se sientan las bases de lo que va a ser la «nueva economía doméstica»; Becker, pionero de dicha escuela, parte de la consideración de que una vez que la información sobre control de natalidad haya alcanzado por igual a todos los grupos de población, la actitud de las parejas familiares ante sus expectativas de fecundidad será idéntica a la de cualquier otro bien de consumo. Así las pautas de consumo (de mercancías y de niños) setán directamente proporcionales a la renta disponible.

Pero, como es lógico, tanto las mercancías como los niños no son considerados sólo desde el punto de vista de la cantidad, sino también de la calidad. Según Becker, la calidad de un hijo está en función de la educación que se le piense dar y, en general, de la cantidad de dinero que se vaya a gastar en él.

La conclusión teórica de Becker es que la renta es función directa del número

${ }^{3}$ DE Miguel, Amando, Diez errores sobre la población española, Madriđ: Tecnos, 1982 , p. 12.

- Frimpman, M., Teotia de los precios, Madrid: Alianza Editorial, 1972, p. 256.

s Schultz, T. Paul, "Introducción a los análisis económicos de la fecundidad", en. Población, Revista de Información Comercial Española, núm. 496, diciembre 1974, página 107. 
de hijos y de su calidad. Es decir, que las familias con mayor renta tendrán un mayor número de hijos y de una calidad superior ${ }^{6}$. Pero Becker pierde de vista que la renta disponible es un solo factor, mientras el número y la calidad de los hijos son dos factores que no pueden solaparse y que el número reducirá la calidad y un incremento en ésta el número de hijos.

En 1962 Friedman realiza nuevos planteamientos dentro del mismo modelo, los hijos suponen no sólo un coste, sino, también, un beneficio, son un bien de consumo comparable a la adquisición de un automóvil u otros bienes de cuyo consumo se obtiene un beneficio por su uso, además son también un bien de capital comparable a la inversión en maquinaria o edificios cuya renta es diferida ${ }^{\text {? }}$.

Estas teorías son eficazmente criticadas en 1968 por Judith Blake, la cual rebate con energía la explicación de que los ricos tendrán más hijos y los pobres menos, por el hecho de conocer por igual los métodos anticonceptivos, por el simple expediente de contrastar esta hipótesis con los datos de todas las sociedades conocidas, en las que para que este modelo se cumpliese, en función de la posibilidad de acceder al conocimiento generalizado de los métodos anticonceptivos, debería producirse un cambio sociocultural radical. Y esto no ha ocurrido, ni es previsible que ocurra en ningún país desarrollado. En conclusión, «aunque la demanda de bienes de consumo duradero se encuentre limitada por el poder adquisitivo, la demanda de hijos no está bajo tal control monetario" "

Estas y otras críticas similares dan lugar a un replanteamiento de las antiguas posiciones de la teoría de la «nueva economía doméstica» de la fecundidad. El propio Becker, modificando sus antiguas posturas economicistas, pasa a planteamientos mucho más sociológicos usando para ello las bases epistemológicas que le proporcionan el interaccionismo, modificando así el concepto de renta que no se cuantifica económicamente sino que es una renta social, individual y subjetivamente valorada cuyos efectos sobre la fecundidad están más en función de la satisfacción personal y familiar que del bienestar económico entendido como un cierto nivel de renta.

A pesar de su acercamiento a la sociología, en 1976 Becker mantiene que: «El enfoque económico proporciona ese marco unificado para la comprensión del comportamiento que buscaron sin resultado tanto Bentham, como Comte, Marx y otros.» Por supuesto, prestando importancia a otras disciplinas como la sociología, psicología, etc. ?.

Mención especial debemos hacer a un representante independiente de las teorías económicas de la fecundidad, se trata de Leibenstein, que establece un modelo explicativo que relaciona los cambios producidos en la fecundidad con el desarrollo

- Becker, G. S., "An Economic analysis of fertility", National Bureau of Economic Research, 1960, pp. 209-231.

7 Frisduna, M., op. cit., pp. 254 y ss.

- Blake, Judith, "Are babies consumer durables? A critique of the Economic theory of Reproductive motivation", Population Studies, vol. XXII, núm. 1, marzo 1968, p. 17.

- Becker, G. S., "El enfoque económico del comportámiento humano", en "Nuevas aplicaciones del análisis económico. El mercado político y la conducta humana", Revista de Información Comercial Española, núm. 557, enero 1980, p. 18. 
económico, diferenciando además los beneficios y los costes que producen los hijos:

\section{«Beneficios}

1. Consumo entendido como satisfacción personal.

2. Trabajo o renta que el niño cuando entre en la fuerza de trabajo aportará a la familia.

3. Seguridad referida al apoyo que el hijo prestará a sus padres cuando éstos sean viejos.

\section{Costes}

1. Coste directo de educación, ropa, comida, etc.

2. Coste indirecto. Pérdida de oportunidades debido al tiempo dedicado al cuidado y educación del niño.

Estos costes $y$ beneficios estarán influenciados principalmente por tres aspectos del desarrollo socioeconómico, que son:

1. Incremento de la renta per cápita.

2. Descenso de la mortalidad.

3. Cambios en la estructura ocupacional.»

Leibenstein excluye de sus hipótesis y trabajos siempre los dos primeros hijos, e inicia sus correlaciones a partir del tercer, cuarto y rangos sucesivos del nacimiento de los hijos ${ }^{10}$.

El autor más prolífico y a su vez más controvertido es, sin lugar a dudas, Easterlin. El modelo que elabora se basa en la teoría de los ciclos de Kuznets, en la que se demuestra la interacción de los fenómenos demográficos con los económicos. Retomando esta teoría, Easterlin plantea la hipótesis de que el tamaño de la cohorte está relacionado negativamente con la fecundidad. La variable fundamental de este modelo es el mercado de trabajo: «Si una cohorte particular es sustancialmente superior a la anterior, esto tendrá una influencia negativa sobre la posición competitiva en el mercado de trabajom ". Esto, a su vez, creará un conflicto entre las aspiraciones y recursos, siendo las aspiraciones el resultado de la «socialización» de los individuos en el domicilio familiar. «Easterlin contempla el comportamiento fecundo como el resultado de una elección familiar en la que los recursos familiares se contrapesan con las preferencias/aspiraciones, lo que, por otro lado, proviene de la experiencia de los jóvenes (en edad de contraer matrimonio y de procrear) en casa de sus padres durante los años previos a su entrada en el mercado de trabajo" ${ }^{12}$.

in LEIBENSTEIN, H.: "An interpretation of the economic theory of fertility: promising path or blind alley?, Quarterly Journal of Economic Literature, 12, pp. 457-479.

"Congdon, Peter, "Forecasting births in greater London: An Application of "the Easterlin Hypothesis", Population Studies, vol. XXXIV, núm. 2, julio 1980, p. 267.

12 Enursch, John, "The relevance of the 'Easterlin Hipothesis' and the 'New Home Economics' to Fertility Movements in Great Britain", Population Studies, vol. XXXIII, número 1, marzo 1979, p. 40. 
Las implicaciones que las afirmaciones anteriores plantean van en dos direcciones, la primera da a la hipótesis un contenido económico que hace referencia al mercado de trabajo. La cohorte o generación que encuentra fácil salida en el mercado de trabajo, por estar compuesta de un número de miembros que no llega a saturar dicho mercado y por lo tanto «le resulta fácil ganar suficiente dinero para establecerse según su estilo deseado de vida por él y su actual o posible novia, se casará y la procreación se verá alentada" ${ }^{13}$. A la generación siguiente, por el contrario, le resultará más duro ganar dinero, por lo que tanto el matrimonio como la procreación se aplazarán.

En este sentido es en el que I. Agüero y A. Olano ${ }^{14}$ han aplicado la hipótesis de Easterlin, utilizando como indicador de las fluctuaciones de la fecundidad la relación entre la población masculina de 30.64 años y la población masculina de 15.29 años, el resultado por ellos obtenido ha sido negativo, en España no es aplicable el modelo de Easterlin.

La segunda implicación, mucho más sociológica, afirma que la experiencia del individuo en su adolescencia antes de abandonar el hogar familiar, será determinante en el modelo de vida deseado para el futuro. Esta ha dado lugar a la acuñación de un nuevo término, «estatus económico relativo", que es el estatus actual del individuo comparado con el de su padre.

En realidad este modelo se ha demostrado insuficiente, como prueban las numerosas variaciones que distintos autores han introducido en el mismo, sin embargo, a efectos de este trabajo, es necesario tenerlo en cuenta por su relación con la sociología de la familia, que bien podría constituir un punto de partida de las investigaciones que desde esta perspectiva se hagan de la fecundidad.

El propio Easterlin, consciente de este acercamiento, realiza un trabajo de síntesis entre la economía y la sociología ${ }^{15}$ que es continuación de los anteriores y trata de resolver el conflicto entre aspiraciones y recursos, que finalmente da lugar a un nuevo modelo ${ }^{16}$ mucho más elaborado, en el que revisa todas sus aportaciones anteriores, introduce variables nuevas y hace especial hincapié en las preferencias endógenas y en la fertilidad. Detallo éste a continuación.

"EAsterlin, R. A., "Relative Economic Status and the American Fertility Swing", en E. B. Sheldon (ed.), Family Economic Behaviour: Problems and Prospects, Toronto and Philadelphia: Lippincott, 1973, en nota 1, p. 181.

"Agüzro, I., y Olano, A., "La evolución reciente de la fecundidad en España", R.E.I.S., núm. 10, abril-junio 1980 , pp. 135 y ss.

is Enstzrlin, R. A.. "The Conflict betwem aspirations and Resources", Population and Development Review, 2, septiembre-diciembre 1976, pp. 417 y ss.

16 Engterlin, R. A.; Pollack, R. A. y Wachter, M. L., "Toward a more general Economic Model of fertility determination: Endogenous preferences and natural fertility", en Ensterlix, R. A., Population and economic change in Developing countries, Chicago: The University Chicago Press, 1980, pp. 81-82. 
«Modelo de los determinantes de la fecundidad marital y tamaño completo de la familia

Los determinantes se analizan a través de las preferencias familiares hacia el consumo, los niños, y la regulación de la fecundidad, por medio de cuatro constricciones:

1. Una presunta constricción que refleja las limitaciones consecuencia de los precios de mercado, de los bienes y servicios, de niveles de sueldos de los miembros de la familia, de cualquier otro tipo de renta, y del tiempo disponible de los miembros de la familia.

2. La tecnología doméstica, que convierte los bienes de mercado y el tiempo de los miembros de la familia en un bienestar básico, lo que es el argumento de su utilidad funcional.

3. Una 'función de nacimientos' o 'función de producción de fecundidad' que expresa el número de nacidos vivos como una función de: frecuencia del coito, intervalo genésico, prácticas reguladoras de la fertilidad, prácticas que presiden la probabilidad de concepción y los periodos no fecundos de la mujer.

4. Una función de la mortalidad infantil, expresada a través de la madurez fisiológica en función de variables, tales como salud y nutrición. Sustrayendo la mortalidad de la fecundidad, se obtendrá el tamaño completo de la familia.»

Muestra especial preocupación en diferenciar dos colectivos diferentes, aquellos en los que se produce un control de la natalidad deliberadamente y aquellos en que este hecho no se produce, lo que no significa que no se produzca control de la natalidad, pero el estudio de la fecundidad debería ser diferente en uno y otro caso.

No voy a entrar en críticas o consideraciones acerca de este complejo modelo, que, por otro lado, no ha sido aplicado todavía; sí quiero, sin embargo, señalar que sigue manteniendo las perspectivas sociológicas que señalaba con anterioridad y que el punto tercero retoma claramente las "variables intermedias" propuestas por Davis para el estudio de la fecundidad, y que veremos a continuación.

Para terminar con las teorías económicas de fecundidad, quiero señalar que términos como "gustos», "deseos», «comportamiento», «decisiones», etc., son de uso corriente, términos que yo considero absolutamente sociológicos. Pero, además, se está produciendo una "relativización» de términos económicos, como es el caso de «estatus económico relativo", ya señalado; el de "coste relativo», acuñado por la "nueva economía doméstica» ${ }^{17}$, que tiene relación con la percepción subjetiva, dependiente del contexto cultural en el que se desenvuelven las familias, "renta relativa», etc.

" Lundert, Peter H., "Child cost and economic development", en EAsterLin, R. A., Population and economic change in developing countries, Chicago y Londres: The University of Chicago Press, 1980. 
Entrando en la perspectiva demográfica, el único modelo elaborado es el de la transición demográfica, cuya principal preocupación es la de explicar el cambio generalizado que se ha producido en las pautas demográficas, el proceso de todos conocido, y que no me detendré a explicar, nos interesa debido a que una de las variables que desempeña un importante papel en el modelo es la fecundidad y concretamente su descenso, que relacionado en un principio con el descenso de la mortalidad, ha dado pie por su insuficiencia explicativa y meramente descriptiva, a numerosas investigaciones. Estas investigaciones tratan de buscar la variable o las variables que inciden sobrẹ la fecundidad, pero trascienden el marco de la demografía y se introducen en el campo de la economía, sociología, etc. Por esto, y dado que la técnica utilizada para la recogida de datos es generalmente sociológica, resulta verdaderamente difícil diferenciar unos trabajos de otros.

La demografía histórica coherente con su propio método, que consiste en realizar su trabajo de campo a través de registros parroquiales, se sitúa en una posición muy relacionada con el tema que nos ocupa.

Es de resaltar la actitud del conocido demógrafo histórico Wrigley, cuando señala cómo todos los acontecimientos demográficos se producen en el seno de la familia modificándola, para añadir que: «Pese a la ocasional ambigüedad que el uso de la palabra familia puede producir, se trata de una unidad de estudio excepcionalmente adecuada” ${ }^{18}$.

Otro demógrafo apoya también esta idea en 1981, Caldwel comenta: «Una correcta teoría de la alta fecundidad y del descenso de la misma será esencialmente una teoría del cambio social y familiar" ${ }^{19}$.

Entrando en las teorías sociológicas, lo primero que llama la atención es la multiplicidad de las hipótesis, relacionando una serie de variables con la fecundidad, aunque podemos avanzar que los resultados han sido positivos sólo parcialmente. Las hipótesis de mayor extensión han dado paso a las encuestas de fecundidad, donde primero se desarrollaron fueron en Estados Unidos. Posteriormente, debido a recomendaciones de las Naciones Unidas, se ha lanzado una Encuesta Mundial de Fecundidad que ya se ha realizado en 23 países. Su objetivo es principalmente a efectos comparativos. En España se pasó en 1977.

El contenido y planteamiento de estas encuestas quedará mejor plasmado si tomamos una de las primeras como ejemplo. La primera que se realizó en Indianápolis dejó varias cuestiones pendientes, que quedaron recogidas en la segunda, la de Princeton. Esta elabora una detallada hipótesis en la que se consideran cuatro variables dependientes, influenciadas por varias variables independientes, siendo las primeras:

1. Deseos personales a partir del tercer embarazo.

2. Disponibilidad psicológica hacia la contracepción.

3. Uso actual y efectividad de la contracepción.

4. Fecundidad actual.

"Wricley, E. A., Historia y población, Madrid: Guadarrama, 1969, p. 11.

19 Caldwell, J. C., op. cit., p. 24. 
Las variables independientes quedan recogidas en tres grupos:

1. Marco social:
a) Ocupación.
b) Educación.
c) Religión, etc.

2. Variables personales:
a) Actitudes.
b) Factores psicológicos, etc.

3. Variables del grupo familiar:
a) Integración conyugal.
b) Dominio hombre-mujer.
c) División familiar del trabajo.
d) Estructura familiar e interacción.

Los resultados de esta encuesta no fueron muy satisfactorios, puesto que lo único que se logró verificar fue la relación entre la religión y la fecundidad, pero se ha de tener en cuenta los defectos metodológicos que dicha encuesta tenía a nivel de ámbito de la muestra, representatividad, etc.

A pesar de estos primeros y relativos fracasos, se siguieron haciendo encuestas de fecundidad en los mismos Estados Unidos y, posteriormente, en el resto de los países del mundo, con lo cual se consiguió ir progresivamente mejorando la metodología y las hipótesis, y llegar así a varias conclusiones que se repetían en las diversas encuestas. Principalmente se detectó la existencia de diferencias en la fecundidad en función de la renta y del hábitat rural o urbano, los dos únicos factores generalizables hasta el momento.

A efectos de este trabajo, creo necesario resaltar que el tercer grupo de variables que constituye la encuesta pertenece al ámbito de la sociología de la familia, y desde ellas podrá explicarse gran parte del comportamiento frente a la fecundidad.

La problemática francesa requiere una mención especial. Pionera en el proceso de la transición demográfica, alcanzó tasas de fecundidad tan bajas que llegaron a preocupar a los Gobiernos sobre el futuro de su población. Como consecuencia de esta especial situación, los enfoques que se han dado en Francia a las encuestas de fecundidad son diferentes. Estas encuestas son básicamente de opinión y en ellas se aprecian dos constantes: la primera, que podríamos definir como la búsqueda de los factores que retraen la fecundidad, y la segunda, la posible aceptación o no de los ciudadanos de una serie de medidas adoptadas o por adoptar que favoreciesen el incremento de la fecundidad.

A continuación, comento una de las encuestas francesas mencionadas, que he seleccionado por ser representativa y por aparecer claramente reflejadas en la misma las constantes antedichas. 
El presupuesto del que parte esta encuesta es la forma en que el nacimiento de un niño afecta las condiciones familiares. Los factores analizados son:

1. El embarazo y el parto pueden tener una incidencia directa sobre la salud de la madre.

2. El crecimiento de la familia modifica las condiciones de alojamiento.

3. El equilibrio del presupuesto familiar se verá afectado.

4. El tiempo libre y de ocio se verá reducido en favor del tiempo empleado en el trabajo doméstico.

5. La actividad profesional de la madre se verá afectada.

La conclusión es que si bien un cierto número de familias se sienten perjudicadas en alguno de estos factores, no los van a tener en cuenta a la hora de decidir su fecundidad, puesto que ésta sigue escapando a la percepción de la investigación e incluso al control de los propios padres. «Sin duda, la percepción de las condiciones de alojamiento, del nivel de recursos o de la compatibilidad entre actividad profesional y responsabilidades familiares, interviene, pero en el contexto general de un modelo en el que. el rol individual parece preeminente. Este modelo colectivo está bajo la dependencia de múltiples y poderosos factores en lenta evolución "20.

Por tanto, cualquier medida que se pueda adoptar relacionada con los factores citados, sólo servirá para mantener la natalidad en el nivel actual, sin que las familias vean degradado su nivel de vida por un nuevo nacimiento deseado, pero nunca se les incitará a tener un hijo suplementario.

El planteamiento del estudio de la fecundidad del que parten los franceses según se puede apreciar, reviste especial alcance en cuanto a la «explicación» de la fecundidad, ya que toman como unidad de análisis el conjunto familiar.

Finalmente, voy a exponer el modelo de Kingsley Davis, que es el primero que se elabora desde una perspectiva sociológica, con una pretensión explicativa. Parte de la existencia de muchos factores sociales que inciden sobre la fecundidad, por lo que tratando de lograr un marco de referencia analítico a través del cual se pueden comparar tanto el descenso de la fecundidad en los países desarrollados como el lento descenso en los países subdesarrollados, elabora una serie de variables intermedias, en las que cualquiera de los factores sociales citados ha de incidir bien positiva o negativamente.

Estas variables intermedias en realidad son las que mantienen una relación directa con la fecundidad.

«I. Factores que afectan la exposición a las relaciones sexuales ('variables de las relaciones sexuales').

A) Los que gobiernan la formación y disolución de uniones en el período reproductivo.

- Collows, Philippe, "La naissance d'un enfant. Incidence sur les conditions de vie des familles", Population, núm. 3, 1977. 
1. Edad en que comienzan las uniones sexuales.

2. Celibato permanente: proporción de mujeres que nunca participan en una unión sexual.

3. Parte del período reproductivo transcurrida después de las uniones, o entre ellas.

a) Cuando las uniones son interrumpidas por el divorcio, la separación o el abandono.

b) Cuando las uniones son disueltas por la muerte del esposo.

B) Los que gobiernan la exposición a las relaciones sexuales dentro de las uniones.

4. Abstinencia voluntaria.

5. Abstinencia involuntaria (por impotencia, enfermedad, separaciones inevitables pero temporales).

6. Frecuencia del coito (excluyendo los períodos de abstinencia).

II. Factores que afectan la exposición a la concepción ('variables de la concepción').

7. Fecundidad o infecundidad, en tanto son afectadas por causas involuntarias.

8. Utilización o no de anticonceptivos.

a) Por medios mecánicos y químicos.

b) Por otros medios.

9. Fecundidad o infecundidad, en tanto son afectadas por causas voluntarias (esterilización, subincisión, tratamiento médico, etc.).

III. Factores que afectan la gestación y el parto con éxito ('variables de la gestación').

10. Mortalidad fetal por causas involuntarias.

11. Mortalidad fetal por causas voluntarias» ${ }^{21}$.

(Nota: Por error de la traducción, donde se lee fecundidad, debe leerse fertilidad.)

Este modelo, total o parcialmente, está presente en la mayoría de los trabajos que se realizan sobre fecundidad, aunque no con ese carácter de intermediario que era la premisa de Davis, sino equiparando estas variables a las variables socioeconómicas que se utilizan.

21 Davis, K., y Buaks, J., "Estructura social y fertilldad: un marco de referencia analítico", en La sociedad humana, Buenos Aires: Eudeba, 1965, pp. 658-659. 
Posteriormente, con relación a la teoría de la transición demográfica, elabora Davis la teoría de la «respuesta polifásica» o de «cambio y respuesta», en la que se expone la hipótesis de que cualquier cambio demográfico obtiene una respuesta que neutralizará los efectos que dicho cambio pudiese producir.

Esta hipótesis trata de explicar el proceso de transición demográfica y más concretamente el crecimiento de la población, como consecuencia del descenso de la mortalidad ${ }^{22}$, la cual producirá varios tipos de respuestas por parte de la población:

1. Migración rural-urbana.

2. Migraciones internacionales.

3. Retrasos en la edad de contraer matrimonio.

4. Celibato.

5. Control de la natalidad,

los cuales, a fin de cuentas, son los responsables del descenso de la fecundidad. Elaborada en un principio para los países industrializados, es aplicada al caso de Puerto Rico de 1940 a $1970^{23}$, única ocasión en que ésta ha sido tenida en cuenta, el interés relativo de la misma es que en última instancia trata de indicar los efectos de la elección de respuesta en la tendencia sobre la tasa de natalidad.

No quiero terminar esta primera parte sin dejar constancia de algunos de los planteamientos que se han barajado en el Congreso General de la Unión Internacional para el Estudio Científico de la Población, celebrado en Manila del 9 al 16 de diciembre de $1981^{24}$.

Gaisie, a partir de un estudio realizado en Ghana, ha constatado grandes diferencias de comportamientos entre subgrupos de una misma población y el interés por tanto de descomponer el análisis de la fecundidad en este sentido. McDonald sugiere que en la sociedad se producen modelos sociales insconscientemente ratificados por las parejas, más que una toma de decisión deliberada fundada en gustos particulares o cálculos racionales, refiriéndose a la edad de contraer matrimonio, negando cualquier posible relación entre la edad de contraer matrimonio y el número de hijos deseado por la pareja.

Otra aportación de interés para el estudio de la fecundidad, es la de Bulatao y Fawcett, en la que textualmente leemos: «Los autores insisten sobre la idea de que el número final de hijos no es el resultado de una decisión tomada de una vez por todas, sino de una secuencia de decisiones que tienen en cuenta, en cada caso, la situación de la familia y los cambios exteriores.» Asimismo, la variedad de estrategias familiares en función de las clases sociales fue expuesta por Torrado.

22 Citado en Andorka, R., Determinants of fertility in advanced Societies, Londres: Methuen and Co., Ltd., 1978, p. 25.

${ }^{23}$ Mosher. W. D., "The Theory of Change and Response: An Application to Puerto Rico, 1940-1970". Population Studies, vol. 34, núm. 1, marzo 1980.

${ }^{24}$ Un resumen completo del Congreso se encuentra en el número 2 de Population, marzo-abril 1982, pp. 247 y ss. Utilizo las ponencias más interesantes a efectos de este artículo. 
En el área de la demografía económica se discutió acerca del problema metodológico de la elección de la unidad de estudio: la familia, el individuo, etc. Federici, apoyándose en la hipótesis de Easterlin, plantea para el caso de la mujer el problema del acoste de oportunidades», y Timur analiza la imposibilidad de estudiar el rol femenino al margen de la célula familiar. Se habla también de roles femeninos y de la posible evolución en las relaciones de pareja hacia una mayor integración o, por el contrario, mayor individualismo. Finalmente, se realizó una mesa redonda sobre «Las funciones familiares y sus consecuencias demográficas».

Resulta interesante apreciar cómo van recogiendo sus frutos algunos de los sociólogos que por los años 60 defendían la premisa de analizar la fecundidad tomando como unidad de estudio el núcleo familiar. Voy a citar textualmente a algunos de ellos que se adelantaron veinte años a los acontecimientos.

Ronald Freedman, en 1963, decía: «Cuando los miembros de una sociedad hacen frente a problemas comunes con importantes consecuencias sociales, tienden a desarrollar una solución normativa. Así, en cada sociedad y grupo de población, existen normas prescribiendo cómo han de ser los modelos de crecimiento de la familia» ${ }^{25}$.

William J. Goode, también en 1963, lo planteaba en los siguientes términos: "La sociología debe admitir que no ha determinado todavía los principales factores que han afectado al cambio de las tasas de natalidad en la sociedad occidental... El sociólogo no debe contentarse con cualquier simple explicación económica... Dentro de un círculo social, hay definiciones específicas sociales y culturales acerca de cuántos hijos son deseables.... ${ }^{26}$.

Por último, citaré a Judith Blake, que en 1968 señalaba: «La teoría de la motivación reproductiva es al mismo tiempo la teoría de la familia y la sociedad ${ }^{27}$

Hasta este punto, he desarrollado una compilación sintética y sistemática de las principales teorías sobre el fenómeno de la fecundidad, las cuales se han desarrollado principalmente fuera de España. La síntesis excluye una profundización en aportaciones empíricas particulares y por tanto su crítica. La filosofía de este artículo es exponer una serie de sugerencias, a partir de las cuales, se podría programar investigaciones empíricas concretas para contrastar la situación española.

Finalmente, en resumen, y desde la perspectiva de la especialización académica, se establecen varias conclusiones que conllevan a diversas consecuencias metotodológicas:

La primera de ellas es que realmente la economía es la disciplina, que en la actualidad se preocupa a nivel internacional por la problemática de la fecundidad, sin embargo, es una ciencia económica evolucionada, con muchas concesiones sociológicas, puesto que por sí misma no lograba avanzar hacia explicaciones causales.

"Frempan, R., "Norms for family size in underveloped areas", Proceedings of the Royal Society, B. 159, 1963.

26 GOoD, W. J., World Revolution and family patterns, The Free Press or Glencoe, 1963, pp. 51 y ss.

27 BLAKE, J., op. cit., p. 24. 
La segunda, es que los economistas españoles no parecen dispuestos a dejarse contaminar por las elucubraciones sociodemográficas de sus colegas anglosajones.

Hemos aprendido también que la demografía se encuentra en una situación delicada respecto a su objeto científico y a sus perspectivas de futuro, lo que parece haberla estancado en una pura metodología cuantificadora.

Por último, debemos concluir con la constatación de que la sociología desempeña un papel cada vez más importante para todos los autores no sociólogos (los economistas y demógrafos especialmente), y, concretamente, al rol estratégico que se le atribuye a la estructura familiar. Sin embargo, los sociólogos, mucho más moderados, intentan establecer un modelo más amplio de relaciones de interdependencia entre las variables que influyen en la fecundidad, aunque no dejan de reconocer su relativo fracaso. La única alternativa causal válida, parece ser la estructura familiar hegemónica en la sociedad o grupo social considerado objeto del estudio.

\section{El caso español}

El primer dato a retener es el atraso o incluso el desconocimiento con que las teorías de la fecundidad llegan a España en comparación con el resto de los países desarrollados que nos rodean. La primera barrera, fácilmente superable, es la falta de traducciones incluso de los trabajos más relevantes.

Entre las causas a las que podemos atribuir la responsabilidad en esta situación, figura el desinterés institucional por los problemas demográficos existentes en España, desinterés que se plasma en una falta de planificación demográfica y que es consecuencia de que nunca se han producido problemas de superpoblación o de descenso alarmante de las tasas de fecundidad.

Sin embargo, y aunque sea de una manera superficial, debo citar los estudios que sobre fecundidad se han realizado en España, los cuales se reparten en tres épocas muy bien delimitadas y bastante aisladas.

La primera época comprende la década de los años 40 y se articula alrededor de la figura de Ros Jimeno, que se ocupa de evaluar las consecuencias de la Guerra Civil y estudiar las perspectivas de la natalidad en España con objeto de superar dicho trauma. La segunda época corresponde a las últimas etapas del desarrollismo y surge impulsada por la posibilidad de poder tratar más o menos abiertamente sobre control de natalidad, el ejemplo más acabado es la encuesta de fecundidad realizada por Juan Díez Nicolás en 1974, a la que hay que añadir otras aportaciones de Salustiano del Campo y Amando de Miguel. Tales trabajos carecen de continuidad por las razones antes expuestas, y la fecundidad no vuelve a ser estudiada hasta que son publicados en 1979 los resultados de la «Encuesta de Fecundidad» de 1977, elaborada por el Instituto Nacional de Estadística, en base a la cual se han publicado algunos trabajos, entre ellos los de Jesús de Miguel y Alonso Hinojal ${ }^{28}$.

26 Véase el número monográfico de la R.E.I.S., núm. 10, dedicado a Población. 
Evidentemente, estamos en un círculo vicioso; como consecuencia de una serie de causas objetivas no hay investigación empírica sobre la fecundidad, lo que impide contrastar los resultados de las hipótesis teóricas enunciadas en otros países, esta falta de contrastación produce un desconocimiento del contenido de dichas teorías, y ese desconocimiento se traduce, finalmente, en un desinterés de los profesionales implicados. Considero que si los profesionales interesados en la fecundidad iniciaran de alguna manera investigaciones sobre la fecundidad, se podría producir una presión institucional con la que podríamos escapar del actual callejón sin salida.

Creo necesario aclarar una serie de conceptos utilizados, aunque se ha demostrado que un excesivo énfasis en la definición de los conceptos redunda negativamente en su eficacia, por lo que voy a describirlos prioritariamente en función de su operatividad. Aquí voy a definir unos pocos conceptos básicos.

Demográficamente, natalidad y fecundidad son dos conceptos bien diferenciados, la natalidad es una tasa bruta entre el total de nacimientos por el total de población. Debido a que esta medición está mediatizada por la estructura de edades de la población y por la proporción entre los sexos, a efectos comparativos se formaliza la tasa de fecundidad que reduce el total de población a las mujeres en edad de procrear. Sociológicamente, sin embargo, la natalidad es el resultado final de la fecundidad, que a su vez es el resultado de la confluencia de dos fenómenos: fertilidad y control de la natalidad.

Así, desde una perspectiva sociológica, debemos distinguir el fenómeno de la natalidad y diferenciarlo de la medida de la fecundidad, y en el momento de establecer correlaciones con otras variables sociales, debemos precisar el concepto que manejamos, ya que las conclusiones pueden ser muy diferentes, según utilicemos datos de natalidad o datos de fecundidad.

Por otro lado, el componente fundamental de los estudios de población es la fecundidad $y$ en muchas ocasiones se reemplaza el análisis poblacional en el que intervienen otras variables, por un simple análisis de la fecundidad, a cuyo modelo se le añade el conjunto de variables que responden al fenómeno de la evolución de las poblaciones. Hay que evitar confusiones, la fecundidad es un fenómeno demográfico, metodológicamente al mismo nivel jerárquico que la mortalidad, las migraciones, etc., y es un error querer convertir estos fenómenos en variables dependientes de la fecundidad, la cual se confunde así con el fenómeno demográfico total.

Otra característica cada vez más preponderante en los estudios de fecundidad es que éstos se centran de forma creciente en la mujer, al referirse las propias tasas exclusivamente a ella, así las encuestas plantean cuestiones en torno a los caracteres de esta mujer; su nivel cultural, actividad laboral, número de hermanos, conocimiento de métodos anticonceptivos, etc. En algunos casos, determinadas variables familiares se han visto reducidas exclusivamente a su componente femenino, como en el caso de «el ciclo vital de la familia», que en última instancia la O.M.S. convierte operativamente en el «ciclo vital de la mujer»" ${ }^{29}$.

29 Der Caxapo, Salustiano, "El ciclo vital de la familia", Sistema, núm. 37, julio 1980, página 83. 
Finalmente, me cabe hacer referencia a ciertas cuestiones relativas a la estructura familiar y que de alguna forma afectan directamente al contenido de las teorías enunciadas. Primeramente estarían las variables «más importantes que afectan internamente la estructura familiar y que son la integración conyugal, las conductas frente a la planificación familiar y la comunicación entre padres e hijos" ${ }^{30}$, que han evolucionado hacia una mayor transparencia, integración e igualdad.

En segundo lugar, el fenómeno del mantenimiento de la estructura de la familia ampliada, confirmado por datos empíricos tanto en Francia ${ }^{31}$, como en España ${ }^{32}$, que en contra de lo afirmado por numerosos especialistas hace algunos años se mantiene, siendo las relaciones más intensas y regulares cuando se habita en la misma ciudad, y más esporádicas pero permanentes en distancias inferiores a 500-600 Km., como consecuencia de mutuas prestaciones de servicios que afectan a la dinámica familiar y concretamente a la fecundidad.

Finalmente, hay que considerar las nuevas formas de familia aparecidas en los últimos años, que de manera creciente han diversificado la forma clásica de familia nuclear o extensa. Estas son ${ }^{33}$ :

\section{A) Forma incompleta de familia conyugal}

1. Celibato.

2. Familia de una cabeza.

3. Matrimonio de hecho.

4. Cohabitación.

5. Matrimonio abierto.

B) Forma de familia conyugal

1. Familia conyugal.

2. Familia de doble carrera.

C) Forma ampliada de familia conyugal

1. Matrimonio de grupo.

2. Diversas formas de comunas.

De todas ellas, a efectos del estudio de la fecundidad, sólo resultan operativas en España: la familia conyugal y la familia de doble carrera.

Una vez realizadas las anteriores aclaraciones, paso definitivamente a exponer las hipótesis que podrían ser contrastadas en el caso español.

30 PICK DE WeIss, S., Un estudio social-psicológico de la planificación familiar, México: Siglo XXI, 1979, p. 49.

"Gokalp, C., "Le reseau familial", Population, núm. 6, noviembre-diciembre 1978, página 1088.

${ }^{32}$ FOESSA, Informe sociológico sobre la situación social de España, Madrid: Euroamérica, 1976.

${ }^{33}$ Alonso Hinosal, I., "Población y familia", R.E.I.S., núm. 10, abril-junio 1980, p. 165. 


\section{Elementos para una bipótesis}

Llegados a este punto, resulta interesante plantear una serie de hipótesis que nunca se han verificado en nuestro país. Estas hipótesis deberán cubrir dos campos complementarios. En primer lugar, se trata de averiguar de dónde proceden las decisiones de tener hijos para pasar posteriormente al análisis de la adopción generalizada de un modelo preponderante en cuanto al número de los mismos.

Los estudios de' fecundidad en paises desarrollados pueden partir de considerar el supuesto de racionalidad weberiano ${ }^{34}$, debido a la posibilidad de controlar la natalidad, cuyo resultado final ha sido un fuerte descenso de la fecundidad, aunque dicho supuesto no se ajusta con el alto porcentaje de «hijos no deseados», que aparecen en las encuestas de los países occidentales.

En el caso español, para situarnos a la altura del resto de Occidente, desde un punto de vista puramente demográfico o de sociología de la población, faltan dos elementos que son: una intensa campaña de información sexual y planificación familiar y la legalización del aborto, como corrector de errores en última instancia.

Sólo en este caso podríamos plantearnos realizar los análisis, al mismo nivel que se realizan en otros países, a pesar de lo cual, el supuesto de racionalidad no es un buen criterio de partida, sin explicaciones complementarias que redefinen su contenido y consecuencias. Entran en juego muchos factores que determinan el comportamiento, cuyos componentes son elementos subjetivos, de oportunidad y del entorno.

En este sentido, me inclino a pensar que los comportamientos en materia de fecundidad operan a un nivel de preconsciente. «Motivaciones no expresadas, bien por olvido, bien porque son encubiertas, pero de las que los demandantes (consumidores) pueden llegar a tomar conciencia, tras vencer una mayor o menor resistencia (fenómeno que de hecho suele ocurrir con frecuencia en la dinámica de las reuniones de grupo $){ }^{35}$.

El medio en el que se producen estas decisiones es de un cambio social profundo referido a valores y normas, en el que lo primero a determinar para el caso del estudio de la fecundidad es la unidad de la que procede la decisión.

En España, la tasa de ilegitimidad sigue siendo baja, por lo que la mayoría de los nacimientos se producen dentro del matrimonio, es decir, dentro del ámbito familiar. Esto nos lleva a la primera de las cuestiones: en los supuestos que exista toma de decisión, ¿quién toma la decisión de tener un hijo, la mujer, el hombre o conjuntamente?; en los supuestos de embarazo no deseado, ¿quién decide su interrupción o continuación? Por supuesto, en última instancia, la mujer tiene la posibilidad de obrar según su propio criterio, sin embargo, no es aceptable pensar que en temas tan importantes la mayoría de las mujeres obren por su cuenta, es más, en la familia tradicional la autoridad del hombre sigue siendo indiscutible.

La coexistencia entre diferentes formas familiares, autoritarias e igualitarias

34 Cicourel Anzon, V., El método y la medida en Sociologia, Madrid: Editora Nacional, 1982.

${ }^{35}$ ORTI, Alfonso, El análisis empirico del comportamiento del consumidor, mimeografiado, 1978, p. 40 . 
en España es un hecho, a la vez que se entremezclan factores de modernización con pautas tradicionales. Se puede observar una mayor tendencia a «la liberalización progresiva de las normas sociales (inter-sexos, padres e hijos...)", a la vez que «... la red de relaciones familiares continúa siendo importante dentro de la trama social, favoreciendo la existencia de particularismos familistas» ${ }^{36}$. Esto hace que no sea fácil de determinar, sin un cuidadoso análisis empírico, todo lo referente a la decisión de tener hijos.

El nivel preconsciente del que hablábamos anteriormente, que Alfonso Orti sitúa como un nivel intermedio entre el consciente y el inconsciente, se elabora a partir de una serie de modelos de actitudes y comportamientos ya existentes, que, sin embargo, no llegan a fijarse $\in \mathrm{n}$ un nivel consciente. Se puede afirmar que lo relativo a la toma de decisión de tener hijos se sitúa en este nivel por la gran dificultad que encuentran un alto número de personas a responder sin ambigüedades por qué tienen uno, dos o más hijos.

Algunos de los factores que contribuyen a la elaboración de modelos a nivel preconsciente, pueden ser: la imitación, las presiones del entorno social y las relaciones afectivas.

La imitación desempeña un papel muy importante en modelos de comportamiento entre miembros de una misma comunidad, tanto entre pequeños grupos como entre países. Los subgrupos familiares (hermanos, padres, etc.), de vecindad, de compañeros de trabajo, etc., a través de un intercambio constante de pareceres, se convierten en modelos a imitar. El proceso de imitación del modelo europeo a través de los factores: turismo, emigración y medios de comunicación de masas, ha sido analizado por Rosa Conde: «... los efectos más directos se producen sobre la familia, con la adopción de nuevas pautas de comportamiento que han favorecido la reducción del número de hijos, el uso de anticonceptivos...." ${ }^{37}$.

La difusión de las ideas, normas y valores que la unidad familiar adopta sobre sí misma y de las que derivan los comportamientos conscientes o preconscientes, resulta, por lo tanto, un factor a tener en cuenta en el estudio de la fecundidad.

Las presiones del entorno social, coartan al individuo tratando de reconducirlo a la norma a través de mecanismos muy simples pero efectivos. En este sentido funcionan argumentos que van desde: lo bueno que es para la salud tener hijos, hasta conocidos refranes como «un hogar sin hijos es como un jardín sin flores». En un reciente análisis cualitativo que realizábamos un equipo de sociólogos sobre la problemática de la mujer, se reflejaba muy efectivamente este elemento coactivo, una de las mujeres en una de las reuniones de grupo lo hacía patente expresándose así: «... te casabas y tu suegra o tu madre como llevaras tres meses sin estar en estado te decían que no valías». La cuestión se plantea, por tanto, en torno al grado de incidencia de estas presiones en los comportamientos procreadores de las parejas.

36 Andrés Orizo, Francisco, Perspectivas de cambio sociocultural en España, mimeografiado, 1980 , pp. 6 y 53.

${ }^{37}$ Conde, Rosa, "Desarrollo económico y cambio familiar: el impacto del nuevo rol femenino sobre la estructura de la familia", en Rosa Conde, Familia y cambio social en España, Madrid: Centro de Investigaciones Sociológicas, 1982, p. 158. 
El último factor son las relaciones afectivas y de proyección que tradicionalmente han sido especialmente relevantes en cuanto a los comportamientos familiares, y que hoy en algunos ámbitos podrían estar cambiando de signo, debido precisamente a la evolución sufrida en las relaciones familiares y en la emancipación de los hijos.

Con independencia de estos tres factores determinantes, no podemos, ni debemos, olvidar la influencia de ciertas variables estructurales sobre la fecundidad, como son: grado de urbanización, nivel de educación, religiosidad, etc.; por el contrario, se deberían incrementar los esfuerzos realizados en este sentido, a la vez que es imprescinditle comenzar a trabajar con las hipótesis expuestas en estas páginas, tratando de profundizar en la búsqueda de relaciones causales $\mathrm{y}$, en definitiva, de una explicación sociológica a la fecundidad. 